Original

\title{
No Modifying Effect of Antioxidant N-Acetyl-L-Cysteine on Fenofibrate-induced Hepatocarcinogenesis in Rats
}

\author{
Jihei Nishimura ${ }^{1,2}$, Yasuaki Dewa ${ }^{1,2}$, Meilan Jin $^{3}$, Yukie Saegusa ${ }^{1,2}$, \\ Masaomi Kawai ${ }^{1,2}$, Sayaka Kemmochi ${ }^{1,2}$, Keisuke Shimamoto ${ }^{1,2}$, \\ Tomoaki Harada ${ }^{1}$, Tadashi Itoh ${ }^{4}$, Tomomi Shima ${ }^{1}$, Makoto Shibutani $^{1}$, and \\ Kunitoshi Mitsumori ${ }^{1}$ \\ ${ }^{1}$ Laboratory of Veterinary Pathology, Tokyo University of Agriculture and Technology, 3-5-8 Saiwai-cho, Fuchu-shi, \\ Tokyo 183-8509, Japan \\ ${ }^{2}$ Pathogenetic Veterinary Science, United Graduate School of Veterinary Sciences, Gifu University, 1-1 Yanagido, Gifu- \\ shi, Gifu 501-1193, Japan \\ 3 Division of Pathology, National Institute of Health Sciences, 18-1 Kamiyoga, Setagaya-ku, Tokyo 158-8501, Japan \\ ${ }^{4}$ Hashima Laboratory, Nihon Bioresearch Inc., 104, 6-Chome, Majima, Fukuju-cho, Hashima, Gifu 501-6251, Japan
}

\begin{abstract}
To clarify the modifying effect of N-Acetyl-L-Cysteine (NAC), which has antioxidative ability, on hepatocarcinogenesis promoted by fenofibrate (FF), a peroxisome proliferator-activated receptor (PPAR) alpha agonist , male F344/N rats were administered a single intraperitoneal injection of $N$-diethylnitrosamine (DEN) as an initiator followed by administration of a diet containing 3,000 ppm of FF for 16 weeks. Two-thirds partial hepatectomy was performed 1 week after the FF treatment. Additionally, NAC treatments for 14 weeks from 2 weeks after the FF treatment were performed. Although the expression level of tumor protein p53 (Tp53) mRNA decreased in the $\mathrm{DEN}+\mathrm{FF}+\mathrm{NAC}$ group as compared with that in the DEN+FF group, no significant differences between the DEN+FF and $\mathrm{DEN}+\mathrm{FF}+\mathrm{NAC}$ groups were observed in the number of hepatocellular altered foci and activities of hepatocellular proliferation. In addition, the results of an antioxidant enzyme assay and measurement of the amounts of total glutathione in the liver revealed no significant difference between the DEN+FF and DEN+FF+NAC groups; although no significant differences were observed in many genes between the DEN+FF and DEN+FF+NAC groups, only glutathione peroxidase 2 (Gpx2) mRNA increased in the DEN+FF+NAC group as compared with the DEN+FF group. The results under the present experimental conditions indicate no obvious modifying effect of NAC on liver tumor promotion by FF in rats. (J Toxicol Pathol 2009; 22: 255-261)
\end{abstract}

Key words: PPAR alpha, fenofibrate, N-Acetyl-L-Cysteine, liver, oxidative stress, hepatocarcinogenesis

\section{Introduction}

Reactive oxygen species (ROS) induce oxidative damage in various cell constituents such as DNA, proteins and lipids through oxidative stress ${ }^{1}$. In particular, oxidative DNA damage causes mutations and abnormal gene expressions that are involved in carcinogenesis ${ }^{2}$. Treatments with antioxidants or free radical scavengers are a powerful technique to investigate the involvement of ROS in chemical-induced acute and chronic injuries and carcinogenesis. Nakae et al..$^{3-5}$ reported that $N$-tert-Butyl- $\alpha$ phenylnitrone (PBN), which is a nitrone-based compound

Received: 18 May 2009, Accepted: 25 June 2009

Mailing address: Kunitoshi Mitsumori, Laboratory of Veterinary

Pathology, Tokyo University of Agriculture and Technology 3-5-8

Saiwai-cho, Fuchu-shi, Tokyo 183-8509, Japan

TEL/FAX: 81-42-367-5771

E-mail: mitsumor@cc.tuat.ac.jp that traps or scavenges free radical species such as $\mathrm{H}_{2} \mathrm{O}_{2}$ and superoxide anion and has been widely used as a spin trapping agent in the detection of free radical species, has inhibitory effects with decreased formation of 8-hydroxyguanine in DNA on hepatocarcinogenesis model in rats fed a cholinedeficient, L-amino acid-defined (CDAA) diet. NishikawaOgawa et al. ${ }^{6}$ reported that the treatments with N-Acetyl-LCysteine (NAC), which is an analogue of cysteine as well as a precursor of reduced glutathione (GSH) and plays a role in enhancing the activities of glutathione S-transferases, glutathione peroxidase, glutathione reductase, $\mathrm{NADH}$ - and NAD(P)H-quinone reductase, inhibited the development of GST-P positive foci in the livers of rats treated with 2amino-3,8-dimethylimidazo[4,5-f]quinoxaline (MeIQx) in the post-initiation stage. NAC has also been reported to decrease significantly the incidences of neoplastic and preneoplastic lesions in many organs, including the liver, induced by a variety of chemical carcinogens in rodents ${ }^{7}$. As 
mentioned above, antioxidants are reported to be useful in a number of ways with regards to inhibition of tumor formation. Some studies have also shown that patients with virus-mediated hepatocellular carcinomas have lower levels of anti-oxidants in their blood and livers ${ }^{8}$.

Fenofibrate (FF), a member of the fibrate class of hypolipidemic drugs, has been extensively used in many countries to treat hypertriglyceridemia and mixed hyperlipidemia ${ }^{9}$. It belongs to the broad class of chemicals known as peroxisome proliferators (PPs), which act through the peroxisome proliferator activated receptor $\alpha$ (PPAR $\alpha)$. Information about FF have been released by the U.S. Food and Drug Administration (FDA) that shows FF to be carcinogenic to rodent species when administered at high doses; $200 \mathrm{mg} / \mathrm{kg}$ of FF administered to rats for 24 months or to mice for 21 months increased the incidence of hepatocellular carcinomas in both sexes. However, FF showed no mutagenic potential in the following four tests: Ames, mouse lymphoma, chromosomal aberration and unscheduled DNA synthesis. Rusyn et al. ${ }^{10}$ have shown that the treatment with Wy-14643, a known rodent nongenotoxic carcinogen and a PP, to mice for 1 month significantly increased the expression levels of base excision DNA repair genes with no change of commonly used indicators such as 8-oxoguanine and abasic sites in the liver DNA of control and Wy-14643-treated mice. We have also recently identified changes that indicate DNA damage, such as elevations of 8-hydroxydeoxyguanosine (8-OHdG) and expression of DNA repair enzymes, in the livers of rats in the early stage of repeated FF toxicity and during the preneoplastic foci formation stage, which is linked to oxidative stress ${ }^{11,12}$. On the other hand, there are some studies reporting little or no change in the markers of oxidative DNA damage (e.g., 8-OHdG) in response to PPs $^{13,14}$.

Therefore, in the present study, to clarify the possible mechanism of the oxidative stress-mediated liver tumor promoting effect in rats given FF in greater detail, we performed preliminary experiments using a two-stage hepatocarcinogenesis model in $\mathrm{N}$-diethylnitrosamine (DEN)-initiated rats given simultaneously both FF and NAC and investigated the modifying effect of NAC on liver tumor promotion induced by FF.

\section{Materials and Methods}

\section{Chemicals}

FF (purity, >99\%) was purchased from Sigma-Aldrich Chemical Co. (St. Louis, MO, USA). NAC (purity, >98\%) and DEN (purity, $>99 \%$ ) were purchased from Wako Pure Chemical Industries (Osaka, Japan) and Tokyo Kasei Kogyo (Tokyo, Japan). All other chemicals were of analytical grade and obtained commercially.

\section{Animals and chemicals}

A total of 8 male 5-week-old F344/N Slc rats were purchased from Japan SLC Inc. (Shizuoka, Japan). The rats

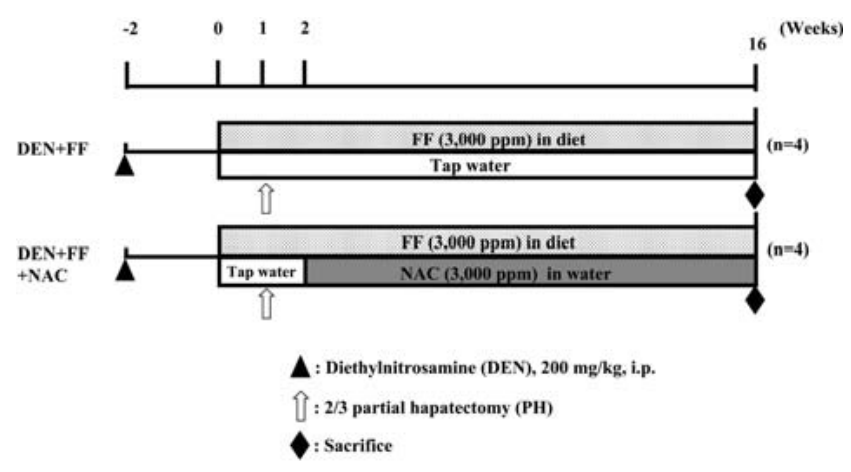

Fig. 1. Experimental design.

were housed in stainless steel cages with 4 animals per cage and allowed ad libitum access to tap water and a commercial powdered basal diet (MF; Oriental Yeast Industries Co., Ltd., Tokyo, Japan). All the animals were handled under standard conditions (room temperature, $23 \pm 3{ }^{\circ} \mathrm{C}$; relative humidity, $55 \pm 15 \%$; 12-h light and dark cycle). The rats were acclimatized for 1 week before treatment with $N$ diethylnitrosamine (DEN; Sigma-Aldrich Chemical Co., St. Louis, MO, USA). Animal care and experiments were carried out in accordance with the Guide for Animal Experimentation of the Tokyo University of Agriculture and Technology.

\section{Experimental design}

The experimental design is shown in Fig. 1. In this preliminary experiment, we used a 2-stage liver carcinogenesis model of rats. After acclimatization, all the animals underwent i.p. injection of DEN (200 mg/kg body weight) dissolved in saline to initiate hepatocarcinogenesis. After 2 weeks, the rats were divided into 2 tests group, consisting of the DEN + FF (4 animals) and DEN + FF + NAC (4 animals) groups, using stratification methods based on body weight on the day before the FF treatment. Beginning on the next day, the groups were fed a diet containing 3,000 ppm FF for 16 weeks; in addition, the DEN $+\mathrm{FF}+$ NAC group was given 3,000 ppm NAC in their water over the period of 2 to 16 weeks after the FF treatment. All rats were subjected to two-thirds partial hepatectomy 1 week after the FF treatment. The dosage of FF in this study was selected based on the results of our previous study ${ }^{12}$. With respect to NAC, Nishikawa-Ogawa et al. demonstrated an inhibitory effect on MeIQx rat hepatocarcinogenesis using NAC at a dosage of $100 \mathrm{mg} / \mathrm{kg}$ body weight by intragastric administration five times/week ${ }^{6}$. In addition, in Swiss albino mice treated with a single i.p. injection of urethane $(1 \mathrm{~g} / \mathrm{kg}$ body weight), administration of 2,000 ppm NAC in the diet (200 mg/kg body weight) significantly decreased both the incidence and multiplicity of lung tumors ${ }^{15}$. In $\mathrm{A} / \mathrm{J}$ mice treated with a single i.p. injection of urethane $(0.25 \mathrm{~g} / \mathrm{kg}$ body weight), administration of 2,000 ppm NAC in the diet significantly decreased the multiplicity of lung tumors ${ }^{16}$. On the other hands, Badawy et al. reported that administration 
of NAC at a dosage of $600 \mathrm{mg} / \mathrm{kg} /$ body weight induced liver dysfunction and damage in adult male rats ${ }^{17}$. Therefore, in the present study, we selected 3,000 ppm NAC as an approximate dosage in drinking water, which is equivalent to $300 \mathrm{mg} / \mathrm{kg}$ body weight $\mathrm{NAC}^{17}$. Body weight, food consumption and water intake were measured once a week. Necropsy was performed under anesthesia with ether at the end of week 8 after fasting for $16 \mathrm{~h}$. All the remaining lobes of the livers were removed and weighed. The liver samples were sectioned, and one section was used for histopathological examinations, while the other sections were frozen in liquid nitrogen and stored at $-80^{\circ} \mathrm{C}$ for future analyses. For light microscopy, formalin-fixed liver tissues were embedded in paraffin, and tissue slices were sectioned. Hematoxylin and eosin (H\&E) staining was performed for the sections in accordance with procedures for the routine histopathological examinations.

\section{Immunohistochemical staining for Ki-67 and GST-P}

Immunohistochemical staining for Ki-67 and glutathione S-transferase placental form (GST-P) was conducted to evaluate cell proliferation and quantitatively analyze of hepatocellular preneoplastic lesions, respectively. Sections for Ki-67 were heated using an autoclave in citrate buffer for antigen retrieval and subjected to immunohistochemistry with a mouse monoclonal antibody against Ki-67 (1:30; clone MIB-5; DAKO Japan, Kyoto, Japan) using the horseradish peroxidase-avidin-biotin complex method and a Histofine SAB-PO Kit (Nichirei, Tokyo, Japan), with 3,3-diaminobenzidine $/ \mathrm{H}_{2} \mathrm{O}_{2}$ as the chromogen. Sections for GST-P were subjected to immunohistochemistry with a rabbit polyclonal antibody against GST-P (1:2000; Medical \& Biological Laboratories, Aichi, Japan) using the horseradish peroxidase-avidin-biotin complex method and a Vectastain Elite ABC kit (Vector Laboratories Inc., Burlingame, CA, USA), with 3,3diaminobenzidine $/ \mathrm{H}_{2} \mathrm{O}_{2}$ as the chromogen. The sections were counterstained with hematoxylin for microscopic observation. The numbers and areas of GST-P positive foci $(>0.2 \mathrm{~mm}$ in diameter) and total areas of the liver sections were measured using the Scion Image software (Scion Corp., Frederick, MD, USA). The labeling index of Ki-67 was expressed as the percentage of positive cells after counting approximately 5,000 hepatocytes in 10 fields $(\times 100)$ selected randomly in each specimen.

\section{Determination of enzyme activities}

The rat liver tissue used for the enzyme assay was homogenized in $10 \mathrm{mM}$ of ice-cold Tris-HCl ( $\mathrm{pH} 7.4)$ containing $0.25 \mathrm{M}$ sucrose and $1 \mathrm{mM}$ EDTA using an allglass Potter Elvehjem homogenizer. Each homogenate was centrifuged for $20 \mathrm{~min}$ at $800 \times g$. The resulting supernatant fraction was used to determine the enzyme activities of carnitine palmitoyltransferase (CPT), fatty acyl-CoA oxidizing system (FAOS), superoxide dismutase (SOD) and catalase. Protein concentrations $(\mathrm{mg} / \mathrm{mL})$ were determined using a BCA Protein Assay Kit (Pierce Biotechnology, IL,
USA). CPT involved in mitochondrial $\beta$-oxidation was measured spectrophotometrically by the methods of Markwell et al. ${ }^{18}$ following the release of a CoA-SH from acetyl-CoA and palmitoyl-CoA each, using the general thiol reagent DTNB (5,5'-dithio-bis-(2-nitrobenzoic acid)). FAOS involved in peroxisomal $\beta$ oxidation was measured spectrophotometrically by the methods of Markwell et al. ${ }^{18}$. Activity was defined as $\mu \mathrm{mol}$ per min per mg protein. The superoxide dismutase (SOD) and catalase levels were determined using an SOD Assay Kit-WST (Dojindo Molecular Technologies, Inc., Gaithersburg, MD, USA) and Amplex Red Catalase Assay Kit (Molecular Probes, Inc., Eugene, OR, USA), respectively, according to the manufacturer's protocol. Activity was defined as units per mg protein.

A liver tissue sample was also deproteinated by the addition of trichloroacetic acid, and DTNB [5,5-dithiobis(2nitrobenzoic acid)] was added to supernatants cleared by centrifugation $(10 \mathrm{~min}, 800 \times \mathrm{g}$ ). The formation of 5-thio-2nitrobenzoic acid, which is proportional to the total GSH concentration, was monitored at $412 \mathrm{~nm}$ against reagent controls $^{19}$. GSH are expressed as mg per g of wet hepatic tissue.

\section{RNA isolation and gene expression analyses}

Total RNA was isolated from 4 animals of each group using TRIzol reagent (Invitrogen Corp., Carlsbad, CA, USA) according to the manufacturer's protocol. Quantitative real-time RT-PCR analyses with the SuperScrip III First-Strand Synthesis System (Invitrogen Corp., Carlsbad, CA, USA) and SYBR green PCR master mix (Applied Biosystems, Foster City, CA, USA) using an ABI Prism 7000 Sequence Detection System (Applied Biosystems) were carried out using the abovementioned 4 RNA samples of each group. Taking into account the finding of our previous reports ${ }^{11,12}$, we assessed the gene expressions of the following 25 genes: acyl-Coenzyme A oxidase 1 (Aco), cytochrome P450, 4A1 (Cyp4a1), acetylcoenzyme A acetyltransferase 1 (Acat1), glutathione peroxidase 2 (Gpx2), growth arrest and DNA-damageinducible 45 alpha (Gadd45a), UDP glycosyltransferase 1 family, polypeptide A6 (Ugt1a6), glutathione S-transferase Yc2 subunit (Yc2), catalase (Cat), glutathione S-transferase, mu 2 (Gstm2), glutathione S-transferase, mu 3 (Gstm3), glutathione-S-transferase, alpha type 2 (Gsta2), cytochrome P450, family 1, subfamily a, polypeptide 2 (Cyp1a2), apurinic/apyrimidinic endonuclease 1 (Apex1), X-ray repair complementing defective repair in Chinese hamster cells 5 (Xrcc5), O-6-methylguanine-DNA methyltransferase (Mgmt), MutL homolog 1 (Mlh1), topoisomerase (DNA) I (Top1), nibrin (Nbn), 8-oxoguanine-DNA-glycosylase (Ogg1), cyclin D1 (Ccnd1), tumor protein p53 (Tp53), cyclin-dependent kinase inhibitor 1B (Cdkn1b), checkpoint kinase 2 homolog (Chek2), growth arrest and DNA-damageinducible 45 beta (Gadd45b) and cyclin-dependent kinase inhibitor 1A (Cdkn1a). To obtain the relative quantitative values for gene expression, $\beta$-actin was used as an 
Table 1. The Effect of Coadministration of NAC on General Findings and the Data for the Liver Weight, GST-P Positive Foci, and Cell Proliferation in the Livers of Rats Given FF after DEN Initiation

\begin{tabular}{|c|c|c|c|}
\hline \multirow{2}{*}{\multicolumn{2}{|c|}{$\begin{array}{l}\text { Groups } \\
\text { Number of rats }\end{array}$}} & $\mathrm{DEN}+\mathrm{FF}$ & $\mathrm{DEN}+\mathrm{FF}+\mathrm{NAC}$ \\
\hline & & 4 & 4 \\
\hline \multicolumn{2}{|l|}{ Final body weight (g) } & $285.0 \pm 12.8$ & $286.3 \pm 17.4$ \\
\hline \multicolumn{2}{|l|}{ Food intake $(\mathrm{g})^{\text {a }}$} & $14.4 \pm 1.2$ & $13.8 \pm 2.3$ \\
\hline \multicolumn{2}{|c|}{ Fenofibrate exposure (mg/kg BW/day) } & $168.4 \pm 20.2$ & $163.1 \pm 23.4$ \\
\hline \multicolumn{2}{|c|}{ Water intake $(\mathrm{mL})^{\text {a }}$} & $20.7 \pm 1.9$ & $18.9 \pm 3.2$ \\
\hline \multicolumn{2}{|c|}{ Antioxidant exposure (mg/kg BW/day) } & 0 & $245.11 \pm 48.4$ \\
\hline \multicolumn{2}{|c|}{ Absolute liver weight (g) } & $19.45 \pm 1.28$ & $19.84 \pm 0.71$ \\
\hline \multicolumn{2}{|l|}{ Relative liver weight (g) } & $6.82 \pm 0.25$ & $6.94 \pm 0.29$ \\
\hline \multirow{3}{*}{$\begin{array}{l}\text { Hepatocellular altered foci } \\
\text { GST-P positive foci }(>0.2 \mathrm{~mm})\end{array}$} & Number (No./cm²) & $10.5 \pm 4.5$ & $10.6 \pm 3.3$ \\
\hline & Area $\left(\mathrm{mm}^{2} / \mathrm{cm}^{2}\right)$ & $0.55 \pm 0.45$ & $0.43 \pm 0.28$ \\
\hline & Number (No./ $\left.\mathrm{cm}^{2}\right)$ & $3.47 \pm 1.42$ & $4.24 \pm 2.50$ \\
\hline \multicolumn{2}{|l|}{ Ki-67 positive cells (\%) } & $1.15 \pm 0.54$ & $2.12 \pm 0.59$ \\
\hline
\end{tabular}

Values are expressed as means \pm S.D. ${ }^{\text {a }}$ Calucuated from the weekly monitoring data.

endogenous control, and its expression levels were calculated according to the $2-{ }^{\Delta \Delta \mathrm{Ct}}$ method $^{20}$. The primers used in the present study were identical to those of our previous studies ${ }^{11,12}$.

\section{Statistical evaluation}

Statistical analyses were performed using statistical software (StatLight; Yukms Co., Ltd., Japan), and all results are presented as means $\pm \mathrm{SD}$. The 2 corresponding groups were compared by analyzing the data using the F-test for homogeneity of variance between the DEN+FF and $\mathrm{DEN}+\mathrm{FF}+\mathrm{NAC}$ groups. If the variance was homogeneous, the student's $t$-test was applied for comparisons, and if it was heterogeneous, the Aspin-Welch's $t$-test was used. A $P$ value of less than 0.05 was considered statistically significant.

\section{Results}

Data for final body weight, food intake, FF intake, water intake, antioxidant intake and liver weight of the rats given FF with or without NAC treatments are shown in Table 1. There was no significant difference in above parameters between the DEN+FF and DEN+FF+NAC groups.

Histopathologically, hepatocellular altered foci were found in the livers of both groups, but their numbers were almost the same in the two groups (Fig. 2). In addition, immunohistochemical examinations for GST-P showed no changes of the number and area of GST-P positive foci between the two groups. There was no significant difference in the activity of hepatocellular proliferation between the $\mathrm{DEN}+\mathrm{FF}$ and DEN+FF+NAC groups (Table 1, Fig. 2).

The results of examination of enzyme activities and the amounts of total GSH are shown in Table 2. The activities of enzymes, such as CPT, FAOS, SOD and catalase, and the amount of total GSH showed no significant differences between the DEN+FF and DEN+FF+NAC groups.
The results of real-time RT-PCR are shown in Table 3. Overexpression of $G p x 2$ mRNA and underexpression of Tp53 mRNA were observed in the $\mathrm{DEN}+\mathrm{FF}+\mathrm{NAC}$ group with statistical significances as compared with the DEN+FF group. The expression levels of other genes in the $\mathrm{DEN}+\mathrm{FF}+\mathrm{NAC}$ group were not significantly different from those in the DEN+FF group.

\section{Discussion}

It is well known that oxidative stress has an important role in chemical carcinogenesis ${ }^{21}$. NAC is a widely used thiol-containing antioxidant that is a precursor of reduced GSH and also affects many ROS-mediated signaling pathways such as c-Jun N-terminal kinase, p38 MAPK, redox-sensitive activator protein-1 and NF- $\kappa B^{22,23}$. With respect to the inhibitory effect of treatment with NAC on liver tumors, Nishikawa-Ogawa et al. reported that intragastric administration of dose of $100 \mathrm{mg} / \mathrm{kg}$ body weight doses of five times/week exerted inhibitory effects on MeIQx-induced rat hepatocarcinogenesis ${ }^{6}$. Therefore, we anticipated that NAC treatment at a dosage of $3000 \mathrm{ppm}$, which is equivalent to $300 \mathrm{mg} / \mathrm{kg}$ body weight NAC and higher than the dose used by Nihikawa-Ogawa et al. ${ }^{6}$, would inhibit tumor-promoting activity via the oxidative stress of FF. However, in the present study, no significant differences were observed in the number of hepatocellular altered foci between the $\mathrm{DEN}+\mathrm{FF}$ and $\mathrm{DEN}+\mathrm{FF}+\mathrm{NAC}$ groups. On the other hand, NAC-induced suppression of oxidationmediated carcinogenesis has been described in some mouse models, such as Atm ${ }^{24}$ and Trp53 knockout mice ${ }^{25}$. Indeed, these mice showed increased ROS production and/or compromised antioxidant defense, but NAC treatment reversed the oxidative DNA damage and the frequency of DNA deletions in them through refinement of these decreased functions. In these experiments, drinking water containing NAC (40 mM NAC) was given to the mice to yield an average dose of $1 \mathrm{~g}$ NAC per kg body weight per 

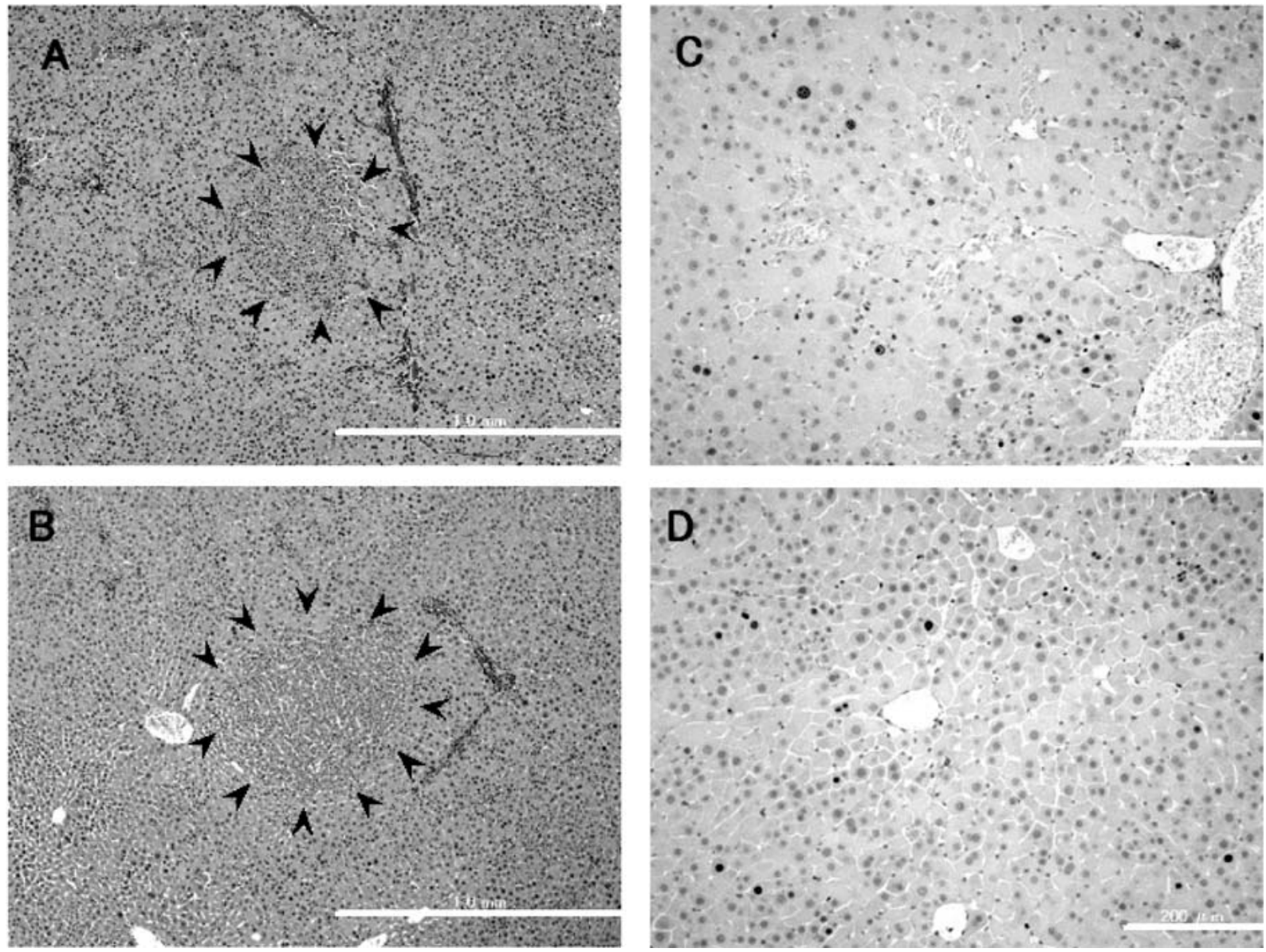

Fig. 2. The effect of coadministration of NAC on the formation of hepatocellular altered foci and cell proliferation in the livers of rats given FF after DEN initiation. A: H\&E staining in the DEN+FF group. Bar=1.0 mm. B: H\&E staining in the $\mathrm{DEN}+\mathrm{FF}+\mathrm{NAC}$ group. Bar=1.0 mm. The area indicated by the arrowheads represents a hepatocellular altered focus. C: Ki67 staining in the DEN+FF group. Bar=200 $\mu \mathrm{m}$. D: Ki-67 staining in the DEN+FF+NAC group. Bar $=200 \mu \mathrm{m}$.

Table 2. The Effect of Coadministration of NAC on Various Enzyme Activities in the Livers of Rats Given FF after DEN Initiation

\begin{tabular}{lcc}
\hline Groups & DEN+FF & DEN+FF+NAC \\
Number of rats & 4 & 4 \\
\hline Carnitine palmitoyltransferase activity $(\mu \mathrm{M} / \mathrm{min} / \mathrm{mg}$ protein) & $1.77 \pm 0.12$ & $1.74 \pm 0.16$ \\
Fatty acid oxidizing system activity $(\mu \mathrm{M} / \mathrm{min} / \mathrm{mg}$ protein) & $3.20 \pm 0.27$ & $3.04 \pm 0.35$ \\
Superoxide dismutase activity (Units/mg protein) & $13.7 \pm 0.7$ & $14.6 \pm 0.9$ \\
Catalase activity (Units/mg protein) & $2074 \pm 173$ & $1966 \pm 378$ \\
Total glutathione (mg/g liver) & $4.2 \pm 1.7$ & $3.0 \pm 0.3$ \\
\hline
\end{tabular}

Values are expressed as means \pm S.D.

day. In addition, the same intake of NAC has been shown to reduce DNA-adduct formation in rats exposed to genotoxic carcinogens and cigarette smoke ${ }^{26}$. Considering these findings, the dose of NAC used in our study may be too low to exert anti-oxidative effects against the tumor promotion of FF in the liver.

With respect to the ROS production by treatment with FF, we have previously reported that FF continuously exerted increased DNA-damaging effects, such as elevation of 8-OHdG in liver DNA, and increased the mRNA levels of DNA repair enzymes in addition to increased fatty acid oxidation and decreased activities of its eliminating enzymes, resulting in enhanced tumor promotion in FFinduced hepatocarcinogenesis ${ }^{11,12}$. Thus, increased ROS production is involved in one of the possible mechanisms of FF-induced hepatocarcinogenesis in rats. However, in the 
Table 3. The Effect of Coadministration of NAC on mRNA Expression Levels in the Livers of Rats Given FF after DEN Initiation

\begin{tabular}{|c|c|c|}
\hline $\begin{array}{l}\text { Functions } \\
\text { Gene name }\end{array}$ & $\begin{array}{c}\mathrm{DEN}+\mathrm{FF} \\
(4)^{\mathrm{a}}\end{array}$ & $\begin{array}{c}\mathrm{DEN}+\mathrm{FF}+\mathrm{NAC} \\
\text { (4) }\end{array}$ \\
\hline \multicolumn{3}{|l|}{ Metabolic stress related genes } \\
\hline Acyl-Coenzyme A oxidase 1 (Aco) & $1.0 \pm 0.2^{\mathrm{b}}$ & $0.8 \pm 0.1$ \\
\hline Cytochrome P450, 4A1 (Cyp4a1) & $1.2 \pm 0.7$ & $1.3 \pm 0.5$ \\
\hline Acetyl-coenzyme A acetyltransferase 1 (Acat1) & $1.0 \pm 0.2$ & $0.8 \pm 0.1$ \\
\hline Glutathione peroxidase 2 (Gpx2) & $1.0 \pm 0.0$ & $1.5 \pm 0.4^{*}$ \\
\hline Growth arrest and DNA-damage-inducible 45 alpha (Gadd45a) & $1.0 \pm 0.2$ & $1.1 \pm 0.4$ \\
\hline UDP glycosyltransferase 1 family, polypeptide A6 (Ugt1a6) & $1.0 \pm 0.2$ & $1.0 \pm 0.4$ \\
\hline Glutathione S-transferase Yc2 subunit (Yc2) & $1.0 \pm 0.1$ & $0.9 \pm 0.4$ \\
\hline Catalase (Cat) & $1.0 \pm 0.3$ & $1.0 \pm 0.4$ \\
\hline Glutathione S-transferase, mu 2 (Gstm2) & $1.0 \pm 0.2$ & $1.0 \pm 0.4$ \\
\hline Glutathione S-transferase, mu 3 (Gstm3) & $1.0 \pm 0.1$ & $1.2 \pm 0.3$ \\
\hline Glutathione-S-transferase, alpha type 2 (Gsta2) & $1.0 \pm 0.1$ & $1.2 \pm 0.4$ \\
\hline Cytochrome P450, family 1, subfamily a, polypeptide 2 (Сyp1a2) & $1.0 \pm 0.4$ & $1.1 \pm 0.3$ \\
\hline \multicolumn{3}{|l|}{ DNA repair related genes } \\
\hline Apurinic/apyrimidinic endonuclease 1 (Apex1) & $1.0 \pm 0.1$ & $0.8 \pm 0.2$ \\
\hline X-ray repair complementing defective repair in Chinese hamster cells 5 (Xrcc5) & $1.0 \pm 0.3$ & $1.1 \pm 0.4$ \\
\hline O-6-methylguanine-DNA methyltransferase (Mgmt) & $1.0 \pm 0.2$ & $0.9 \pm 0.3$ \\
\hline MutL homolog 1 (Mlh1) & $1.0 \pm 0.2$ & $0.8 \pm 0.2$ \\
\hline Topoisomerase (DNA) I (Top1) & $1.0 \pm 0.3$ & $0.8 \pm 0.2$ \\
\hline Nibrin $(N b n)$ & $1.0 \pm 0.4$ & $0.9 \pm 0.3$ \\
\hline 8-Oxoguanine-DNA-glycosylase (Ogg1) & $1.0 \pm 0.1$ & $1.0 \pm 0.3$ \\
\hline \multicolumn{3}{|l|}{ Cell cycle, apoptosis and cell proliferation related genes } \\
\hline Cyclin D1 (Ccnd1) & $1.0 \pm 0.1$ & $1.0 \pm 0.2$ \\
\hline Tumor protein p53 (Tp53) & $1.0 \pm 0.1$ & $0.6 \pm 0.1^{* * *}$ \\
\hline Cyclin-dependent kinase inhibitor 1B (Cdkn1b) & $1.0 \pm 0.1$ & $0.8 \pm 0.2$ \\
\hline Checkpoint kinase 2 homolog (Chek2) & $1.0 \pm 0.1$ & $1.1 \pm 0.3$ \\
\hline Growth arrest and DNA-damage-inducible 45 beta (Gadd45b) & $1.0 \pm 0.1$ & $1.1 \pm 0.3$ \\
\hline Cyclin-dependent kinase inhibitor 1A (Cdkn1a) & $1.0 \pm 0.1$ & $1.3 \pm 0.3$ \\
\hline
\end{tabular}

a Number of rats examined. ${ }^{\mathrm{b}}$ Values of mRNA expression levels (normalized by $\beta$-actin) are expressed as means \pm S.D. The mRNA expression levels are calculated according to the 2 -ddCt method and normalized by $\beta$-actin as an endogenous control. *, ***: $\mathrm{p}<0.05$ and $\mathrm{p}<0.001$, respectively; significantly different from the DEN-FF group, as determined by the Student's $t$-test.

present study, although the change observed in the $\mathrm{DEN}+\mathrm{FF}+\mathrm{NAC}$ group was a significant, but slight, increase in the expression level of Gpx2 mRNA, there were no significant changes in the expression levels of other metabolic stress- and DNA repair-related genes nor in the activities of antioxidant enzymes and amount of total GSH in the liver in this group. Additionally, supplementation of NAC in the promotion stage of FF produced no significant differences in the activity of tumor promotion between the $\mathrm{DEN}+\mathrm{FF}$ and DEN+FF+NAC groups, although a decreased expression level of Tp53 mRNA was observed in the $\mathrm{DEN}+\mathrm{FF}+\mathrm{NAC}$ group as compared with the DEN+FF group. It is known that GPX2, which is a potent detoxifier of ROS, is up-regulated in cancer cells and colitis, and intestinal cancers are induced in $G p \times 1 / G p \times 2$ double knockout mice ${ }^{27}$. In cells having TP53, DNA damageinducing treatment results in a rapid accumulation of TP53 protein $^{28,29}$, which can lead to induction of apoptosis. Yan et al. reported that up-regulation of GPX2 inhibits activation of TP53 by reducing the extent of oxidative stresses and oxidative stress-induced apoptosis in a p53-dependent manner ${ }^{30}$. Considering these findings, the increased $G p x 2$ and decreased Tp53 mRNA observed in the present study may indicate the reduction of oxidative stresses, although the reduction is slight. The reason why NAC treatment was performed from 2 weeks after the FF treatments in our study (or 1 week after the $\mathrm{PH}$ ) was that this design could omit the influences of NAC on the liver regeneration induced after $\mathrm{PH}$ so that we could evaluate only the anti-promoting effects of NAC on liver tumor promotion by FF. Additional studies using higher doses of NAC may show inhibitory effects of NAC on FF-induced hepatocarcinogenesis.

In conclusion, our data from the present study did not demonstrate an obvious inhibitory effect of NAC on liver tumor promotion in rats induced by FF. This finding suggests that the anti-oxidative activity of the NAC used in the present study may be too low to exert anti-oxidative effects against tumor promotion by FF in the liver. Additional investigation with a different experimental design is now in progress to clarify further the possible mechanism of tumor promotion in FF-induced hepatocarcinogenesis in rats.

\section{References}

1. Dröge W. Free radicals in the physiological control of cell 
function. Physiol Rev 82: 47-95. 2002.

2. Marnett LJ. Oxyradicals and DNA damage. Carcinogenesis. 21: 361-370. 2000.

3. Nakae D, Kotake Y, Kishida H, Hensley KL, Denda A, Kobayashi Y, Kitayama W, Tsujiuchi T, Sang H, Stewart CA, Tabatabaie T, Floyd RA, and Konishi Y. Inhibition by phenyl N-tert-butyl nitrone of early phase carcinogenesis in the livers of rats fed a choline-deficient, L-amino aciddefined diet. Cancer Res. 58: 4548-4551. 1998.

4. Nakae D, Kishida H, Enami T, Konishi Y, Hensley KL, Floyd RA, and Kotake Y. Effects of phenyl N-tert-butyl nitrone and its derivatives on the early phase of hepatocarcinogenesis in rats fed a choline-deficient, Lamino acid-defined diet. Cancer Sci. 94: 26-31. 2003.

5. Nakae D, Uematsu F, Kishida H, Kusuoka O, Katsuda S, Yoshida M, Takahashi M, Maekawa A, Denda A, Konishi Y, Kotake Y, and Floyd RA. Inhibition of the development of hepatocellular carcinomas by phenyl N-tert-butyl nitrone in rats fed with a choline-deficient, L-amino acid-defined diet. Cancer Lett. 206: 1-13. 2004.

6. Nishikawa-Ogawa M, Wanibuchi H, Morimura K, Kinoshita A, Nishikawa T, Hayashi S, Yano Y, and Fukushima S. Nacetylcysteine and S-methylcysteine inhibit MeIQx rat hepatocarcinogenesis in the post-initiation stage. Carcinogenesis. 27: 982-988. 2006.

7. De Flora S, Cesarone CF, Balansky RM, Albini A, D’Agostini F, Bennicelli C, Bagnasco M, Camoirano A, Scatolini L, and Rovida A. Chemopreventive properties and mechanisms of $\mathrm{N}$-acetylcysteine. The experimental background. J Cell Biochem. Suppl. 22: 33-41. 1995.

8. Lee KT, Tsai SM, Wang SN, Lin SK, Wu SH, Chuang SC, Wu SH, Ma H, and Tsai LY. Glutathione status in the blood and tissues of patients with virus-originated hepatocellular carcinoma. Clin Biochem. 40: 1157-1162. 2007.

9. Staels B, Dallongeville J, Auwerx J, Schoonjans K, Leitersdorf E, and Fruchart JC. Mechanism of action of fibrates on lipid and lipoprotein metabolism. Circulation. 98: 2088-2093. 1998.

10. Rusyn I, Asakura S, Pachkowski B, Bradford BU, Denissenko MF, Peters JM, Holland SM, Reddy JK, Cunningham ML, and Swenberg JA. Expression of base excision DNA repair genes is a sensitive biomarker for in vivo detection of chemical-induced chronic oxidative stress: identification of the molecular source of radicals responsible for DNA damage by peroxisome proliferators. Cancer Res. 64: 1050-1057. 2004.

11. Nishimura J, Dewa $Y$, Muguruma M, Kuroiwa $Y$, Yasuno H, Shima T, Jin M, Takahashi M, Umemura T, and Mitsumori $\mathrm{K}$. Effect of fenofibrate on oxidative DNA damage and on gene expression related to cell proliferation and apoptosis in rats. Toxicol Sci. 97: 44-54. 2007.

12. Nishimura J, Dewa Y, Okamura T, Muguruma M, Jin M, Saegusa Y, Umemura T, and Mitsumori K. Possible involvement of oxidative stress in fenofibrate-induced hepatocarcinogenesis in rats. Arch Toxicol. 82: 641-654. 2008.

13. Cattley RC, DeLuca J, Elcombe C, Fenner-Crisp P, Lake BG., Marsman DS, Pastoor TA, Popp JA, Robinson DE, Schwetz B, Tugwood J, and Wahli W. Do peroxisome proliferating compounds pose a hepatocarcinogenic hazard to humans? Regul Toxicol Pharmacol. 27: 47-60. 1998

14. Elliott BM and Elcombe CR. Lack of DNA damage or lipid peroxidation measured in vivo in the rat liver following treatment with peroxisomal proliferators. Carcinogenesis. 8: 1213-1218. 1987.

15. De Flora S, Astengo M, Serra D, and Bennicelli C. Inhibition of urethan-induced lung tumors in mice by dietary N-acetylcysteine. Cancer Lett. 32: 235-241. 1986.

16. Balansky $\mathrm{R}$ and De Flora S. Chemoprevention by Nacetylcysteine of urethane-induced lung tumors in mice, as related to the time-course monitoring of micronuclei in peripheral blood erythrocytes. Int J Cancer. 77: 302-305. 1998.

17. Badawy AH, Abdel Aal SF, and Samour SA. Liver injury associated with $\mathrm{N}$-acetylcysteine administration. J Egypt Soc Parasitol. 19: 563-571. 1989.

18. Markwell MA, McGroarty EJ, Bieber LL, and Tolbert NE. The subcellular distribution of carnitine acyltransferases in mammalian liver and kidney. A new peroxisomal enzyme. J Biol Chem. 248: 3426-3432. 1973.

19. Ellman GL. Tissue sulfhydryl groups. Arch Biochem Biophys. 8: 70-77. 1959.

20. Livak KJ and Schmittgen TD. Analysis of relative gene expression data using real-time quantitative PCR and the 2 (Delta Delta C(T)) Method. Methods. 25: 402-408. 2001.

21. Trush MA and Kensler TW. An overview of the relationship between oxidative stress and chemical carcinogenesis. Free Radic Biol Med. 10: 201-209. 1991.

22. Kamata H, Manabe T, Kakuta J, Oka S, and Hirata H. Multiple redox regulation of the cellular signaling system linked to AP-1 and NFkappaB: effects of N-acetylcysteine and $\mathrm{H}_{2} \mathrm{O}_{2}$ on the receptor tyrosine kinases, the MAP kinase cascade, and IkappaB kinases. Ann NY Acad Sci. 973: 419422. 2002.

23. Zafarullah M, Li WQ, Sylvester J, and Ahmad M. Molecular mechanisms of N-acetylcysteine actions. Cell Mol Life Sci. 60: 6-20. 2003

24. Reliene R, Fischer E, and Schiestl RH. Effect of N-acetyl cysteine on oxidative DNA damage and the frequency of DNA deletions in atm-deficient mice. Cancer Res. 64: 51485153. 2004.

25. Sablina AA, Budanov AV, Ilyinskaya GV, Agapova LS, Kravchenko JE, and Chumakov PM. The antioxidant function of the p53 tumor suppressor. Nat Med. 11: 13061313. 2005.

26. Balansky R, Izzotti A, Scatolini L, D’Agostini F, and De Flora $\mathrm{S}$. Induction by carcinogens and chemoprevention by $\mathrm{N}$-acetylcysteine of adducts to mitochondrial DNA in rat organs. Cancer Res. 56: 1642-1647, 1996.

27. Chu FF, Esworthy RS, Chu PG, Longmate JA, Huycke MM, Wilczynski S, and Doroshow JH. Bacteria-induced intestinal cancer in mice with disrupted Gpx1 and Gpx2 genes. Cancer Res. 64: 962-968. 2004.

28. Levine AJ, Momand J, and Finlay CA. The p53 tumour suppressor gene. Nature. 351: 453-456. 1991.

29. Oren M. p53: the ultimate tumor suppressor gene? FASEB J. 6: 3169-3176. 1992.

30. Yan $\mathrm{W}$ and Chen X. GPX2, a direct target of p63, inhibits oxidative stress-induced apoptosis in a p53-dependent manner. J Biol Chem. 281: 7856-7862. 2006. 